\title{
Magnetic fields in classical Be stars
}

\author{
Ruslan Yudin ${ }^{1}$, S. Hubrig ${ }^{2}$, M. Pogodin ${ }^{1}$, I. Savanov ${ }^{3}$, M. Schöller ${ }^{4}$, \\ G. Peters ${ }^{5}$ and M. Cure ${ }^{6}$ \\ ${ }^{1}$ Pulkovo Observatory, St.-Petersburg, Russia; \\ email: ruslan61@hotmail.com; ${ }^{2}$ ESO, Santiago, Chile; ${ }^{3}$ Institute of Astronomy, Russian \\ Academy of Science, Moscow, Russia ${ }^{4}$ ESO, Garching, Germany; ${ }^{5}$ Univ. Southern California, \\ USA; ${ }^{6}$ Univ. de Valparaiso, Chile
}

\begin{abstract}
We report the results of our study of magnetic fields in a sample of Be stars using spectropolarimetric data obtained at the European Southern Observatory with the multi-mode instrument FORS 1 installed at the $8 \mathrm{~m}$ Kueyen telescope. The detected magnetic fields are rather weak, not stronger than $\sim 150 \mathrm{G}$. A few classical Be stars display cyclic variability of the magnetic field with periods of tens of minutes.
\end{abstract}

Keywords. Stars: Be - polarization - magnetic fields

\section{Introduction}

Over an extended period of years many attempts to obtain reliable direct measurements of magnetic fields of Be stars have been rather unsuccessful. Recently, weak field detections in a few Be stars were reported by Hubrig et al. (2007). Below, we report some preliminary results of our most recent observing runs with the multi-mode instrument FORS1 installed at the $8 \mathrm{~m}$ Kueyen telescope at the VLT. We also present first results of our magnetic field time series in the Be star $\lambda$ Eri.

\section{Observations}

As it was already reported by Hubrig et al. (2007), weak photospheric magnetic fields have been detected in four stars, HD 56014, HD 148184, HD 155806, and HD 181615. We also pointed out the likely presence of distinct circular polarisation features in the circumstellar components of Ca II H\&K in the three stars, HD 58011, HD 117357, and HD 181615, indicating a probable presence of magnetic fields in the circumstellar mass loss disks. The FORS 1 observations of HD 181615 are presented in the left panel of Fig. 1. New observations of classical Be stars have been been carried out at the VLT from 2006 to 2008. The Be stars have been observed in 2006 in service mode with the GRISM 600B in the wavelength range $348-589 \mathrm{~nm}$ and with the GRISM 1200B in the spectral range $373-497 \mathrm{~nm}$. The last observations we report here have been carried out in visitor mode in 2008 in the framework of the study of 15 B-type members of the open cluster NGC 3766.

\section{Results}

Weak magnetic fields have been detected in eight classical Be stars. The magnetic field strengths of the studied Be stars are found to be in the range $40 \mathrm{G}$ to $150 \mathrm{G}$. A few Be stars with detected magnetic fields show non-detections at some other observing dates, indicating possible temporal variability of their magnetic fields. To confirm this suggestion we performed time-resolved magnetic field measurements over one hour for a 

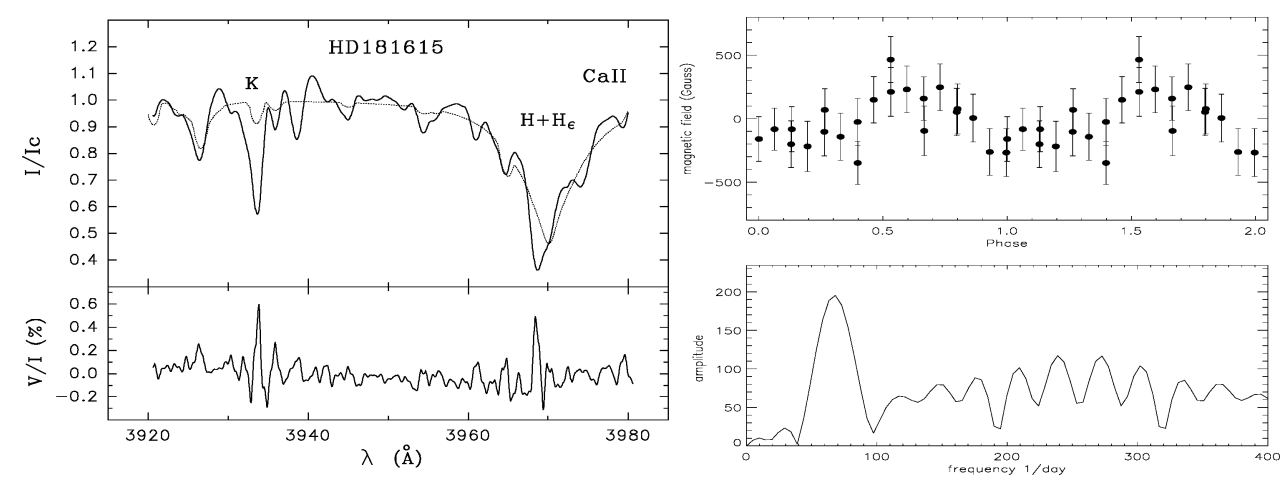

Figure 1. Left panel: Stokes $I$ and $V$ spectra in HD 181615; Right panels: Cyclic variability of the magnetic field in $\lambda$ Eri with a period of $21.1 \mathrm{~min}$ and Fourier spectrum for the magnetic field data derived from hydrogen lines.

Table1. Measurements of NGC 3766 cluster stars with detected magnetic fields.

\begin{tabular}{cccc}
\hline NGC3766 & MJD & $\left\langle B_{z}\right\rangle_{\text {all }}$ & $\left\langle B_{z}\right\rangle_{\mathrm{hydr}}$ \\
\hline 45 & 54550.066 & $-78 \pm 41$ & $-194 \pm 62$ \\
47 & 54549.020 & $-146 \pm 43$ & $-129 \pm 58$ \\
94 & 54550.327 & $294 \pm 53$ & $310 \pm 65$ \\
$\mathbf{1 1 1}$ & 54549.020 & $\mathbf{1 1 2} \pm \mathbf{3 4}$ & $89 \pm 38$ \\
170 & 54550.186 & $1522 \pm 34$ & $1559 \pm 38$ \\
$\mathbf{1 7 6}$ & 54550.016 & $\mathbf{1 2 1} \pm \mathbf{3 6}$ & $\mathbf{1 4 1} \pm \mathbf{4 1}$ \\
$\mathbf{2 0 0}$ & 54550.375 & $\mathbf{1 2 8} \pm \mathbf{4 0}$ & $\mathbf{1 1 5} \pm \mathbf{3 8}$ \\
\hline
\end{tabular}

few Be stars to get information about the behaviour of the magnetic field over at least a part of the stellar surface. The service mode observations revealed periodic changes of magnetic fields on the time scale of tens of minutes in a few classical Be stars. Among these stars, the star $\lambda$ Eri displays a cyclic variability of the magnetic field with a period of $21.1 \mathrm{~min}$ (see the right panel of Fig. 1). The results of the study of fifteen early Btype members of the open cluster NGC 3766 have already been reported by McSwain (2008) who noted two definite and two possible detections in the studied sample. We detected three additional stars with weak magnetic fields which are members of this cluster (highlighted by bold face in Table 1).

\section{Conclusions}

Our search for magnetic fields in Be stars revealed that their magnetic fields are rather weak, but fields of less than $\sim 150 \mathrm{G}$ are not rare. The magnetic fields are clearly variable and a non-detection of magnetic fields in some stars may be explained by temporal variability. A few classical Be stars display cyclic variability of the magnetic field with periods of tens of minutes. The cluster NGC 3766 seems to be extremely interesting, where we find clear evidence for the presence of a magnetic field in seven early B-type cluster members out of fifteen members.

\section{References}

Hubrig, S., Yudin, R., Pogodin, M., Schöller, M., \& Peters, G. 2007, AN 238, 1133

McSwain, M. V., 2008, ApJ 686, 1269 